\title{
Effectiveness of continuous hypertonic saline infusion with an automated infusion pump for decompressive neuroplasty: a randomized clinical trial
}

\author{
Ho-Jin Lee ${ }^{1}$, Jaewoo Lee ${ }^{1}$, Yeon wook Park ${ }^{1}$, Ho Young Gil ${ }^{2}$, Eunjoo Choi ${ }^{1}$, Francis Sahngun Nahm ${ }^{1,3}$, \\ and Pyung Bok Lee ${ }^{1,3}$ \\ ${ }^{1}$ Department of Anesthesiology and Pain Medicine, Seoul National University Bundang Hospital, Seongnam, Korea \\ ${ }^{2}$ Department of Anesthesiology and Pain Medicine, Ajou University Hospital, Suwon, Korea \\ ${ }^{3}$ Department of Anesthesiology and Pain Medicine, Seoul National University College of Medicine, Seoul, Korea
}

Background: Hypertonic saline (HS) injections for decompressive neuroplasty (DN) can cause pain. We assessed whether a continuous infusion of HS through an infusion pump would reduce injection-related pain compared with repeated bolus administrations.

\begin{abstract}
Methods: Fifty patients scheduled for DN were randomized to either the bolus injection or the continuous infusion group. After appropriately placing the epidural catheter, $4 \mathrm{~mL}$ of $5 \% \mathrm{NaCl}$ was injected as four boluses of $1 \mathrm{~mL}$ each at 15-minute intervals or infused over 1 hour using an infusion pump. The severity of pain induced by HS injection, as measured by the 11-point numerical rating scale (NRS), was the primary outcome. The severity of low back or lower extremity pain, as measured by the 11-point NRS and Oswestry Disability Index (ODI), 3 months following the procedure, was the secondary outcome.
\end{abstract}

Results: Data from 21 patients in the bolus group and 23 in the continuous infusion group were analyzed. No statistically significant difference in injection-related pain was identified between the two groups during the initial HS administration $(P=0.846)$. However, there was a statistically significant reduction in injection-related pain in the continuous infusion group compared to the bolus injection group from the second assessment onwards $(P=0.001,<0.001$, and $<0.001$, respectively). No significant between-group differences in the NRS and ODI scores 3 months post-procedure were noted $(P=0.614$ and 0.949 , respectively).

Conclusions: Our study suggests that administering HS through a continuous infusion is a useful modality for reducing HS injection-related pain during DN. (Korean J Pain 2019; 32: 196-205)

Key Words: Chronic Pain; Infusion Pumps; Injections, Epidural; Low Back Pain; Pain, Procedural; Radiculopathy; Saline Solutions, Hypertonic; Spinal Stenosis.

Received January 31, 2019. Revised March 12, 2019. Accepted March 26, 2019.

Correspondence to: Pyung Bok Lee

Department of Anesthesiology and Pain Medicine, Seoul National University Bundang Hospital, 82 Gumi-ro 173beon-gil, Bundang-gu, Seongnam 13620, Korea

Tel: +82-31-787-7499, Fax: +82-31-787-4063, E-mail: painfree@snubh.org

ORCID: https://orcid.org/0000-0003-0325-3356

Ho-Jin Lee's current affiliation: Department of Anesthesiology and Pain Medicine, Seoul National University Hospital, Seoul, Korea

Previous presentation at conferences: The 65th Korean Pain Society Scientific Meeting \& Training Course, 18th Nov. 2017, Venue Grand Hilton Hotel Convention Center, Seoul, Korea.

(a) This is an open-access article distributed under the terms of the Creative Commons Attribution Non-Commercial License (http:// creativecommons.org/licenses/by-nc/4.0/), which permits unrestricted non-commercial use, distribution, and reproduction in any medium, provided the original work is properly cited.

(C) The Korean Pain Society, 2019 


\section{INTRODUCTION}

Decompressive neuroplasty (DN) is an effective treatment for low back pain or lower limb pain caused by adhesions in the epidural space that are refractory to conservative treatment [1]. Adhesions in the epidural space play an important role in chronic lumbar pain syndrome [2-4]. These adhesions occur due to fibrosis of the epidural tissue that is induced during the healing process following tissue damage from spinal surgery or spine degeneration-induced inflammation [3]. Multiple systematic reviews have confirmed that DN is an effective treatment for failed back surgery syndrome, spinal stenosis, and radiculopathy [5-7]. Several drugs, in appropriate volumes, may be administered through the catheter to achieve physical and chemical adhesiolysis.

Among the various drugs used for DN, hypertonic saline (HS) is known to reduce adhesions by removing water from the epidural space through osmosis [8]. Furthermore, HS has cytotoxic effects on fibrous tissue, which might aid in the lysis of epidural fibrosis [9]. Manchikanti et al. [10] showed significant clinical improvement with the epidural administration of HS for adhesiolysis. However, the epidural administration of HS can result in pain during injection [11]. Indeed, it has been reported that the administration of HS stimulates C-fiber afferents [12], and HS is widely used as an experimental model of muscle or other connective tissue pain in humans $[13,14]$. Such findings suggest that HS may stimulate nociceptive afferents in the epidural space, thus causing pain.

To reduce injection-related pain and discomfort, local anesthetics are administered epidurally prior to the administration of HS [11]. However, our previous study using different concentrations of HS revealed that even at lower HS concentrations $(5 \% \mathrm{NaCl})$, mild to moderate injection-related pain was observed, despite the prior administration of a local anesthetic solution into the epidural space [15]. Therefore, in addition to administering a local anesthetic, Jankovic and Peng [16] recommended that HS be administered as an epidural infusion over the course of 15 to 30 minutes.

Although the pain caused by epidural injections of HS is well established, no studies to date have evaluated the effectiveness of various methods for reducing injectionrelated pain. Therefore, the aim of this study was to investigate whether administering a continuous infusion of HS through an infusion pump would reduce the pain compared with that experienced during repeated HS bolus injections.
We expected that, compared with bolus injections, continuous infusion of HS through an infusion pump would reduce injection-related pain by preventing a rapid increase in epidural pressure and reducing the spread of HS.

\section{MATERIALS AND METHODS}

\section{Study participants}

This study was approved by the Seoul National University Bundang Hospital Institutional Review Board of (IRB No: B-1708/415-303) and registered at cris.nih.go.kr (KCT 0003321). The study participants included consecutive outpatients scheduled for DN at our pain center between October and December 2017. In our center, DN is a common procedure performed in patients with back pain or lower limb pain in whom conservative therapies, including epidural steroid injection, have failed.

The main indications for DN that result in such discomfort are spinal stenosis, disc herniation, and failed back surgery syndrome. The inclusion criteria were as follows: (1) age between 18 and 80 years; (2) presence of chronic low back pain, lower extremity radiating pain, or both, for at least 6 months; (3) treatment involving DN using a 1-day protocol including HS infusion; and (4) a pain score of 6 or more on the 11-point numerical rating scale (NRS) after appropriate conservative treatment, including oral analgesics, physical therapy, and epidural steroid injection. The exclusion criteria were as follows: (1) lack of correlation between neuropathic back pain with radicular symptoms and magnetic resonance imaging (MRI) findings; (2) lumbar surgical intervention in the previous 6 months; (3) coagulopathy, chronic infection, or skin infection at the procedure site; (4) poorly controlled psychiatric disorders or acute medical illness, or underlying systemic diseases that could interfere with the interpretation of the outcome assessments; (5) pregnancy or lactation; (6) history of adverse reaction to local anesthetics or steroids; (7) inability to understand informed consent and the study protocol; and/or (8) inability to lie prone for the procedure.

After obtaining written informed consent, eligible $\mathrm{pa}-$ tients were randomly assigned to one of two groups, the bolus injection group (group B) or the continuous infusion group (group C), at the last outpatient session before the procedure. Because the target number of subjects was fewer than 100, block randomization (block size: two or 
four) was performed using a computer-generated randomization program, which was operated by a clinician who was not involved in the study [17].

We recorded the demographic and clinical characteristics of the patients, including age, sex, height, weight, pain scores relating to low back or lower limb pain based on the NRS, duration of pain, the Oswestry Disability Index (ODI) (score range, 0-100), history of lumbar spine surgery, diagnosis based on patient history, pre-procedural MRI findings, current medications, target level of the lumbar spine, and underlying diseases (hypertension, diabetes mellitus, and major depressive disorders).

\section{Procedures}

All procedures were performed by three pain specialists, each with more than 5 years of experience in pain medicine, in an operating room under sterile conditions using fluoroscopic guidance. An intravenous line was established, and cefazolin $(1 \mathrm{~g})$ was administered prior to the start of the procedure. After MRI confirmation of the correlation between the location of the pathology and the radicular pain, $5 \mathrm{~mL}$ of $1 \%$ lidocaine was injected around the sacral hiatus, with the patient lying in the prone position. An 18-gauge Tuohy needle (Sewoon Medical Co., Ltd., Seoul, Korea) was inserted through the sacral hiatus and advanced to the mid-body of the third sacral vertebra under fluoroscopic guidance. After confirming that the needle was properly positioned in the epidural space, lumbar epidurography was performed, with approximately 2 to $5 \mathrm{~mL}$ of Omnipaque ${ }^{\circledR} 300$ (iohexol, 300 mg iodine per $\mathrm{mL}$; GE Healthcare, Piscataway, NJ) as the contrast medium, and filling defects were identified by fluoroscopic imaging [18]. We determined the target of the intervention by correlating the patient's symptoms and MRI or computed tomography findings with the filling defect identified on the epidurogram. If symptoms were present on both right and left sides, the treatment area was designated as the area where the patient had more severe symptoms.

Following epidurography, a 19-gauge epidural catheter (EpiStim $^{\circledR}$; Sewoon Medical Co., Ltd.) with a radiopaque guidewire was slowly passed through a Tuohy needle to the target area. The guidewire was removed and the location of the tip of the epidural catheter was confirmed by injecting contrast medium into the anterolateral epidural space. Mechanical adhesiolysis was performed with 10 to $20 \mathrm{~mL}$ of normal saline. After adhesiolysis, adequate filling of the target nerve roots and epidural space was confirmed. We also confirmed that no accidental intravascular, subarachnoid, or extra-epidural injection had occurred. The final position of the catheter tip was determined via the slow injection of a contrast medium and $6 \mathrm{~mL}$ of $0.25 \%$ ropivacaine (Ropiva injection ${ }^{\circledR}$; Hanlim Pharm Co., Seoul, Korea) containing 1,500 units of hyaluronidase (H-lase ${ }^{\circledR}$; Kuhnil Pharm Co., Seoul, Korea). Following completion of the injection, the catheter was taped utilizing a bio-occlusive dressing, and the patient was placed in a supine position and transferred to the recovery room.

In the recovery room, patients were monitored for any potential complications, including motor weakness over the lower extremities and reduced sphincter tone. After confirming the absence of complications, $4 \mathrm{~mL}$ of $5 \% \mathrm{NaCl}$ solution (Sodium chloride injection ${ }^{\circledR}$; Choongwae Co., Seoul, Korea) [15] was injected according to the following methods. In group B, four bolus injections containing $1 \mathrm{~mL}$ of $5 \% \mathrm{NaCl}$ solution each were administered via the epidural catheter over 5-10 seconds, at 15-minute intervals. In group C, $4 \mathrm{~mL}$ of $5 \% \mathrm{NaCl}$ solution was administered as an infusion over a period of 60 minutes via an infusion pump connected to the epidural catheter. We used a piezoelectric actuated infusion pump (HPMF ${ }^{\circledR}$; Hyun Medics Co., Bucheon, Korea) [19]. This device is used to automatically inject a liquid chemical using a microcomputer, with a piezoelectric actuator as the driving source. Using this device, the drug can be infused over a fixed time interval; a piezoelectric actuated infusion pump is simpler in structure, less faulty, and cheaper than a motor-operated pump, and can be controlled to improve the reliability of the infusion pump with a microcomputer [19]. In addition, hydrophobic filters may be used to prevent the air remaining inside the fluid bag from flowing into the body.

The researchers who performed the procedures, and outcome assessments were blinded to the patients' group assignments. The HS was administered by an anesthesiology resident who was not involved in the study; the anesthesiology resident also measured the residual volume of HS to confirm precise administration of HS into the epidural space through the infusion pump. After the injection of $\mathrm{HS}, 2 \mathrm{~mL}$ of $0.9 \% \mathrm{NaCl}$ solution containing $5 \mathrm{mg}$ of dexamethasone was injected through the epidural catheter, and the catheter was removed. All patients enrolled in this study received a one-day regimen of $\mathrm{DN}$, as described 
previously [10]. Patients were hospitalized on the day of the procedure, given an ambulatory protocol, and discharged after removal of the epidural catheter. Acetaminophen or non-steroidal anti-inflammatory drugs were prescribed as appropriate for pain control during the postprocedure follow-up period.

\section{Outcome measures and follow-up}

All baseline and post-procedure outcome data were collected by a physician at the pain center who was not involved with the study and who was blinded to the study design. The primary outcome of this study was the difference in the intensity of HS-induced pain between the two groups, as scored according to the $11-$ point NRS $(0=$ no pain, 10 = unbearable pain). Infusion-related pain was defined as pain experienced during the epidural HS administration, which was perceived by the patient as different from the baseline lower back or leg pain. Pain assessments were conducted in the recovery room according to the 11-point NRS. In group B, pain scores were recorded during each of the four bolus injections (15-minute intervals); in group C, pain scores were recorded every 15 minutes on four occasions during the 1 -hour infusion period. Pain scores were also recorded at 1 and 3 months after the procedure using the NRS for lower back pain or lower extremity pain during outpatient follow-up. Moreover, the ODI was recorded at the 3 -month follow-up examination. Patients were instructed to report any adverse events, including paresthesia, neuralgia, numbness, and motor weakness, to the physician during the procedure and at each follow-up visit.

\section{Statistical analysis}

A two-arm pilot study that included 19 patients in group $\mathrm{B}$ and 13 patients in group $\mathrm{C}$ was performed before this study. The sample size for the present study was calculated based on the results of the pilot study (group B: $5.11 \pm$ 3.01, group C: $2.31 \pm 2.84$ ). The common standard deviation used was 3.01 , the standard deviation of the bolus group, to ensure a conservative calculation. The formula used to calculate the number of subjects for the study was as follows:

$$
\begin{aligned}
& n_{A}=k n_{B} \text { and } n_{B}=\left(1+\frac{1}{k}\right)\left(\sigma \frac{z_{1-\frac{a}{2}}+z_{1-\beta}}{\mu_{A}-\mu_{B}}\right)^{2} \\
& 1-\beta=\phi\left(z-z_{1-\frac{\alpha}{2}}\right)+\phi\left(-z-z_{1-\frac{\alpha}{2}}\right), z=\frac{\mu_{A}-\mu_{B}}{\sigma \sqrt{\frac{1}{n_{A}}+\frac{1}{n_{B}}}}
\end{aligned}
$$

(k: matching ratio, $\sigma:$ standard deviation, $\alpha$ : Type I error, $\beta$ : Type II error, $\varphi$ : standard normal distribution function)

Based on the pilot study, for $80 \%$ power and a two-tailed significance level of 5\%, 19 patients were required in each study group, for a total sample size of 40 participants. Assuming 20\% dropout, 25 patients were required in each group.

All statistical analyses were performed using R statistical software (ver. 3.2.3; R Foundation for Statistical Computing, Vienna, Austria). Data are presented as the mean \pm the standard deviation, or as the number and percentage of total participants. To compare the demographic and clinical characteristics between the two groups, $t$-tests, $\chi^{2}$ tests, and Fisher's exact tests were used. As the primary outcome measures were obtained at four points in time, a repeated measures analysis of variance was performed with post hoc analyses using $t$-tests. The NRS and ODI scores, which were secondary outcomes, were also analyzed using repeated measures analyses of variance, and $t$-tests were used for post hoc analyses. Within each group, time-to-time comparisons were performed with pairwise comparisons of the predictive margins. The level of statistical significance was set at $P<0.05$.

\section{RESULTS}

Fifty patients were enrolled in the study; 44 completed the full follow-up protocol (Fig. 1). Four patients from group $\mathrm{B}$ and two patients from group $\mathrm{C}$ refused the procedure or withdrew consent to participate in the study. No patients withdrew during the follow-up period. Thus, data from 44 participants (21 in group B and 23 in group C) were included in the final analysis. Table 1 describes the demographic and clinical characteristics of the patients. No significant differences in the demographic and clinical variables were identified between the two groups. 


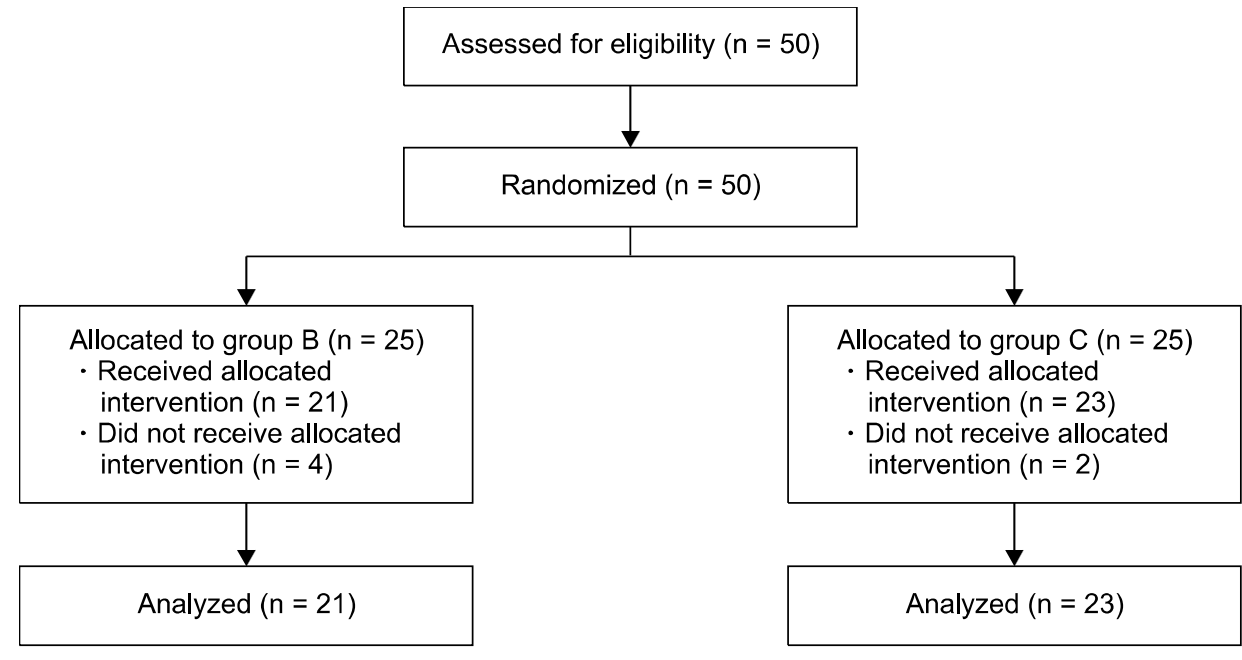

Fig. 1. Flow diagram of patients in this study. At 3 months post-procedure, 21 and 23 patients remained in each arm, respectively.
No statistically significant difference in the injectionrelated pain scores was found between the two groups for the initial administration of HS (Table 2, Fig. 2; $P=0.836$ ). However, there was a statistically significant reduction in injection-related pain in group $\mathrm{C}$ compared with that in group B from the second bolus injection of HS $(P=0.004$, $<0.001$, and $<0.001$, respectively). According to the within-group post hoc analyses, group $\mathrm{C}$ did not show a significant change in the pain scores between the first and subsequent assessments. However, in group B, compared with the first administration, increased pain was noted during subsequent injections (Table 2, $P=0.001,<0.001$, and $<0.001$, respectively). In addition, within-group comparisons for group B demonstrated a significant increase in pain from the second administration to the fourth administration $(P=0.01)$.

During the follow-up period, the NRS scores decreased significantly until 3 months after the procedure compared with the baseline values in both groups (Fig. 3; group B: $P<0.001$, group C: $P<0.001)$. However, no significant differences in the NRS scores were observed between the two groups at the $1-$ and $3-$ month follow-up evaluations ( $P=0.897$ and 0.614, respectively).

The ODI decreased significantly compared with the baseline values until 3 months after the procedure in both groups (Fig. 4; group B: $P<0.001$, group C: $P<0.001$ ). However, no significant difference in the ODI was identified between the two groups after 3 months $(P=0.949)$.

No serious complications during injection or cases of inappropriate drug delivery were reported. No post-procedure complications (hypotension, paresthesia, motor weak- ness, or headache) were reported during the follow-up period, and no other complications, such as infection, sensory deficits, and deterioration of motor function, were reported throughout the study period. No patients withdrew from the study due to adverse effects.

\section{DISCUSSION}

To the best of our knowledge, this is the first study to investigate two different methods of administering $\mathrm{HS}$ for DN and their effects on injection-related pain. The present study demonstrated a statistically significant reduction in the pain experienced by patients who received the HS through an infusion pump relative to those who received the HS through repeated bolus injections for DN.

In this study, patients experienced less pain when HS was infused over 60 minutes through an infusion pump than when HS was administered through four bolus injections. This suggests that the rate of HS administration influenced the severity of injection-related pain. Although the relationship between the rate of HS infusion and pain has not been determined, a study investigating the relationship between local anesthetic-induced pain and the rate of administration reported that the rate of administration had a greater effect on perceived pain during lidocaine infiltration than did buffering [20]. The authors speculated that a slower injection rate was associated with decreased pain levels due to the slower distention of local tissue and the activation of fewer nerve endings. Although we could not measure the actual spread of HS, the slow infusion of HS may prevent it from spreading beyond the 
Table 1. Comparison of the Demographic and Clinical Variables between the Bolus Injection and Continuous Infusion Groups

\begin{tabular}{|c|c|c|c|}
\hline Variable & Bolus injection group $(n=21)$ & Continuous infusion group $(n=23)$ & $P$ value \\
\hline Age (yr) & $70.7 \pm 6.6$ & $68.0 \pm 11.3$ & 0.339 \\
\hline Sex $(M / F)$ & $10(47.6) / 11(52.4)$ & $11(47.8) / 12(52.2)$ & 1.000 \\
\hline Height $(\mathrm{cm})$ & $159.1 \pm 10.7$ & $160.9 \pm 9.9$ & 0.580 \\
\hline Weight $(\mathrm{kg})$ & $63.6 \pm 9.3$ & $67.2 \pm 15.2$ & 0.345 \\
\hline Duration of pain (mo) & $82.1 \pm 99.8$ & $80.8 \pm 115.4$ & 0.968 \\
\hline HTN (yes) & $11(52.4)$ & $16(69.6)$ & 0.390 \\
\hline DM (yes) & $6(28.6)$ & $6(26.1)$ & 1.000 \\
\hline MDD (yes) & $1(4.8)$ & $2(8.7)$ & 1.000 \\
\hline Current medication & & & 0.794 \\
\hline No analgesic use & $3(14.3)$ & $4(17.4)$ & \\
\hline Strong opioid analgesic use $\mathrm{a}^{\mathrm{a}}$ & $4(19.0)$ & $2(8.7)$ & \\
\hline Weak opioid analgesic use only ${ }^{b}$ & $3(14.3)$ & $4(17.4)$ & \\
\hline Non-opioid analgesic use only ${ }^{c}$ & $11(52.4)$ & $13(56.5)$ & \\
\hline Number of previous interventions ${ }^{d}$ & $2.1 \pm 2.3$ & $3.1 \pm 2.6$ & 0.169 \\
\hline Diagnosis & & & 0.279 \\
\hline HIVD & $10(47.6)$ & $6(26.1)$ & \\
\hline Spinal stenosis combined & $8(38.1)$ & $14(60.9)$ & \\
\hline FBSS & $3(14.3)$ & $3(13.0)$ & \\
\hline Stenosis severity ${ }^{\mathrm{e}}$ & & & 0.325 \\
\hline None or mild & $10(47.6)$ & $6(26.1)$ & \\
\hline Moderate & $1(4.8)$ & $2(8.7)$ & \\
\hline Severe & $10(47.6)$ & $15(65.2)$ & \\
\hline Target level & & & 0.129 \\
\hline L3/4 & $0(0)$ & $1(4.3)$ & \\
\hline $\mathrm{L} 4 / 5$ & $5(23.8)$ & $11(47.8)$ & \\
\hline L5/S1 & $16(76.2)$ & $11(47.8)$ & \\
\hline Bilateral symptom & $2(9.5)$ & $1(4.3)$ & 0.935 \\
\hline NRS $(0-10)$ & $7.8 \pm 1.0$ & $7.4 \pm 1.1$ & 0.247 \\
\hline ODI $(0-100)$ & $39.6 \pm 13.3$ & $40.4 \pm 11.7$ & 0.830 \\
\hline
\end{tabular}

Values are presented as mean \pm standard deviation or number (\%).

M: male, F: female, HTN: hypertension, DM: diabetes mellitus, MDD: major depression disorder, HIVD: herniated intervertebral disc, FBSS: failed back surgery syndrome, NRS: numeric rating scale, ODI: Oswestry Disability Index.

${ }^{a}$ Strong opioid analgesics: oral morphine, oxycodone, hydromorphone, and/or fentanyl transdermal patch; ${ }^{b}$ Weak opioid analgesics: tramadol; ${ }^{\circ}$ Non-opioid analgesics: acetaminophen, non-steroidal anti-inflammatory drugs; ${ }^{d}$ Previous interventions included interlaminal epidural block, medial branch block, and transforaminal epidural block. ${ }^{\mathrm{e}}$ The severity of stenosis was determined by the magnetic resonance imaging findings of target level read by the radiologist.

segment in which the local anesthetics had previously been applied, reducing nociceptor irritation.

Another possible mechanism for HS-related pain is an increase in epidural pressure during the injection of HS. A human study reported that the injection speed is significantly correlated with the peak epidural pressure [21]. The peak epidural pressure has also been directly correlated with the speed of injection in an animal study [22]. In addition, it has been reported that the degenerative changes that occur with increasing age decrease the elasticity of the epidural space, leading to higher epidural pressures [23]. The most common indications for DN include degenerative diseases, which are common in elderly patients, wherein the catheter is placed in the most stenotic region. Furthermore, HS is hyperosmolar and is hypothesized to increase the epidural pressure through the transfer of intracellular fluid to the extracellular space. Under these conditions, as HS is injected into the epidural space with reduced elasticity and structural narrowing, the pressure may temporarily increase, resulting in pain caused by compression of the surrounding tissues. Furthermore, the injection of large volumes of fluid can compress nerves 
Table 2. Procedure-related Pain during Hypertonic Saline Injection and Clinical Outcome after Decompressive Neuroplasty

\begin{tabular}{|c|c|c|c|c|}
\hline \multicolumn{2}{|c|}{ Variable } & \multirow{2}{*}{$\begin{array}{c}\text { Bolus injection group }(n=21) \\
\qquad 1.52(0.87-2.18)\end{array}$} & \multirow{2}{*}{$\begin{array}{c}\text { Continuous infusion group }(n=23) \\
\qquad 1.43(0.81-2.06)\end{array}$} & \multirow{2}{*}{$\begin{array}{r}P \text { value }^{a} \\
0.836\end{array}$} \\
\hline Injection NRS (0-10) & 1st injection & & & \\
\hline & 2nd injection & $2.67^{\circ}(2.01-3.32)$ & $1.09(0.46-1.71)$ & 0.004 \\
\hline & 3rd injection & $2.90^{c}(2.25-3.56)$ & $1.13(0.50-1.76)$ & $<0.001$ \\
\hline & 4th injection & $3.57^{\mathrm{c}, \mathrm{d}}(2.97-4.23)$ & $1.04(0.48-1.67)$ & $<0.001$ \\
\hline & Group effect $P$ value $^{b}$ & & & 0.0001 \\
\hline \multirow[t]{4}{*}{ NRS $(0-10)$} & Baseline & $7.81(6.92-8.70)$ & $7.43(6.58-8.29)$ & 0.548 \\
\hline & 1 month & $5.57^{\circ}(4.68-6.46)$ & $5.65^{\mathrm{C}}(4.80-6.50)$ & 0.897 \\
\hline & 3 months & $5.38^{\mathrm{C}}(4.49-6.27)$ & $5.70^{\mathrm{C}}(4.85-6.56)$ & 0.614 \\
\hline & Group effect $P$ value $^{b}$ & & & 0.9892 \\
\hline \multirow[t]{3}{*}{ ODI $(0 \%-100 \%)$} & Baseline & $39.62(34.46-44.77)$ & $40.43(35.51-45.36)$ & 0.821 \\
\hline & 3 months & $25.33^{c}(20.18-30.49)$ & $25.57^{\mathrm{c}}(20.64-30.49)$ & 0.949 \\
\hline & Group effect $P$ value $^{b}$ & & & 0.8768 \\
\hline
\end{tabular}

Values are presented as mean (95\% confidence interval).

NRS: numeric rating scale, ODI: Oswestry Disability Index.

${ }^{a} P$ value in repeated measures analysis of variance testing the contrast effect of treatment at each time; ${ }^{b} P$ value in repeated measures analysis of variance testing the main effect. 'Significant at $P<0.05$ compared to the 1 st injection (Injection NRS) or baseline (NRS, ODI). 'Significant at $P<0.05$ compared to the 2nd injection (Injection NRS).

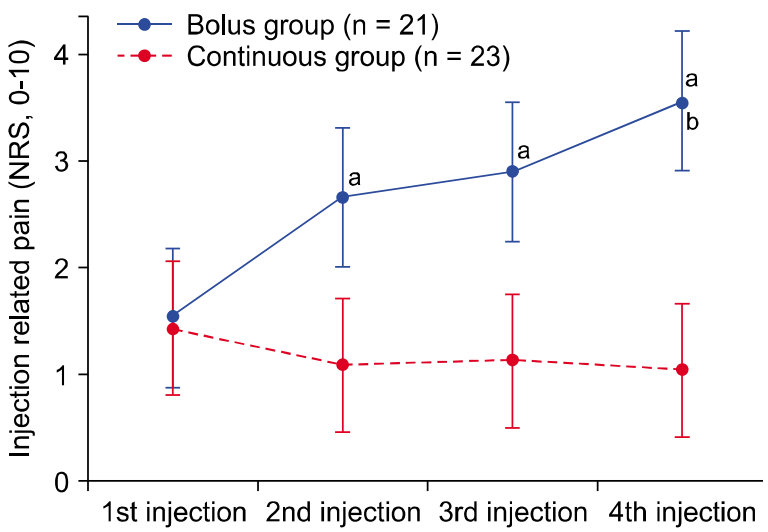

Fig. 2. The 11 -point numeric rating scale (NRS) scores $(0=$ no pain, $10=$ unbearable pain) of injection-related pain induced by hypertonic saline infusion. Data are shown in a box plot with the $95 \%$ confidence intervals (whiskers). ${ }^{\text {a }}$ Significant at $P<0.05$, compared to the 1 st injection NRS. 'Significant at $P<0.05$, compared to the 2nd injection NRS.

and cause transient nerve damage [24]. We think that administering HS through an infusion pump will help to avoid this complication by preventing a sudden increase in epidural pressure.

Another remarkable finding of the study was the increased incidence of injection-related pain associated with an increased number of doses in the bolus group. It was difficult to identify the mechanism underlying these phe-

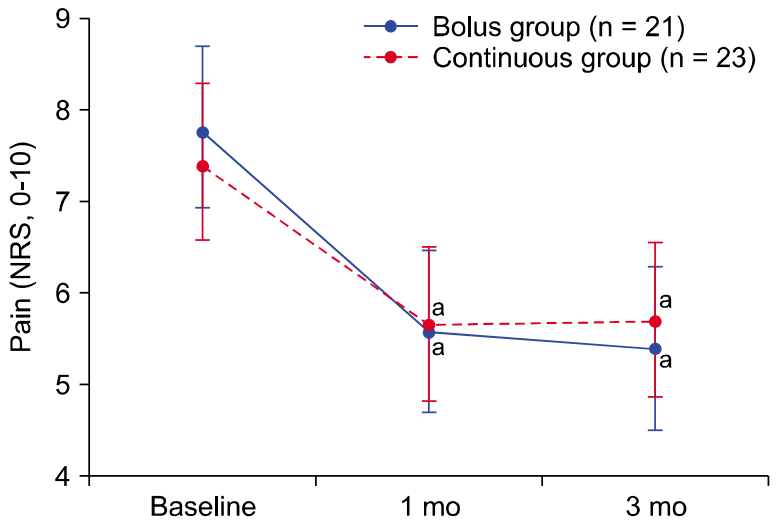

Fig. 3. The 11-point numeric rating scale (NRS) scores (0 $=$ no pain, $10=$ unbearable pain) for the leg and lower back pain in patients receiving lumbar epidural adhesiolysis. Data are shown in a box plot with the 95\% confidence intervals (whiskers). ${ }^{a}$ Significant at $P<0.05$, compared to the baseline NRS.

nomenon in the present study; we assumed that it was partially caused by central hyperexcitability due to repeated injections. A prior study of healthy adult men demonstrated that pain increased as $5 \%$ HS was injected repeatedly within muscles at regular intervals [25]. Although the temporal summation caused by repetitive stimuli in the epidural space is unknown, we suspect that the two methods of administration (i.e., bolus injection with a fixed time interval, and continuous infusion) may have had different 


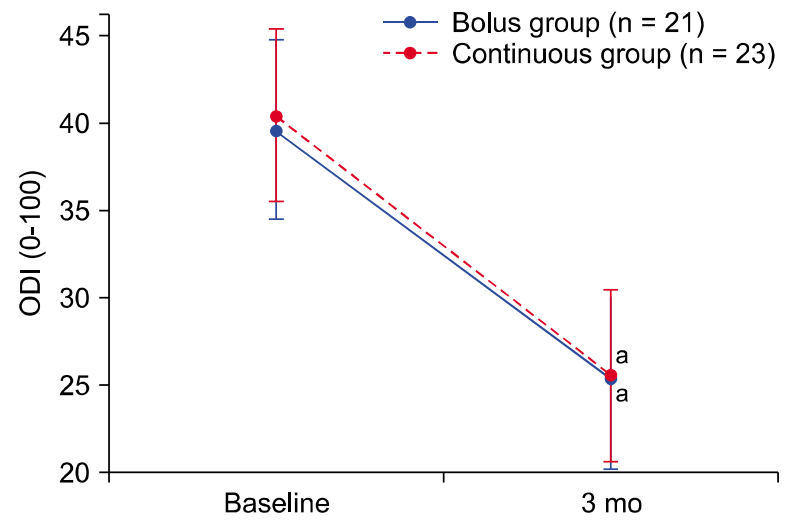

Fig. 4. Oswestry Disability Index (ODI) scores in patients receiving lumbar epidural adhesiolysis. Data are shown in a box plot with the 95\% confidence intervals (whiskers). asignificant at $P<0.05$, compared to the baseline ODI.

effects on the sensitivities of nociception. Further research is needed regarding this phenomenon.

In the present study, we expected the HS to be more concentrated in the target area when infused continuously and slowly, thereby improving the effectiveness of DN. However, no differences in treatment outcomes were noted between the two groups in this study. Although the number of subjects in this study was sufficient to compare injection-related pain between the two administration methods, the sample size was too small to compare the treatment outcomes of DN, which were likely affected by several variables, such as the amount of spinal stenosis. Indeed, although there were no statistically significant differences between the two groups, more spinal stenosis was observed in group $\mathrm{C}$ than in group B. Spinal stenosis is reportedly a poor prognostic predictor of DN [26]. Therefore, to compare the treatment outcomes between the two methods, additional studies that take into consideration the various factors that may affect the outcome of $\mathrm{DN}$ are needed.

Finally, it is less labor-intensive to administer HS as a pump-driven infusion than it is to administer HS as repeated bolus administrations. Bolus administrations need to be divided into several slow injections to reduce injection-related pain, making the procedure cumbersome. The reduction in required labor is particularly important if several patients require treatment on the same day. In our pain center, patients undergo HS administration through an infusion pump over the course of an hour. Physicians do not need to be present during the infusion, as nurses monitor the patients. Thus, in addition to reducing injection-related pain, the administration of HS as an infusion makes the procedure less labor-intensive.

Our study has several limitations. First, the study design precluded patient and doctor blinding. Therefore, we utilized an evaluator who was not involved with the study to assess infusion-related pain. However, we cannot completely exclude placebo effects due to differences in the methods of administration. Second, we did not measure epidural pressures or evaluate the drug distribution within the epidural space. Notably, we could not ignore differences in the epidural pressure and degree of local anesthetic concentration and spread that occurred as a result of the different volumes of contrast medium and normal saline that were administered to each patient. In addition, although the small sample size resulted in a lack of statistical differences in clinical variables between the two groups, we found differences in target level, diagnosis, and severity between the two groups. However, such differences support our findings, because there was greater stenosis in the continuous group, which could lead to increased injection-related pressure that limited drug distribution. Further studies are needed to determine the relationship between the infusion rate and epidural pressures, as well as the relationship between the infusion rate and drug distribution. Third, the infusion pump should reliably deliver a precise volume into the epidural space. In this regard, we also measured the residual volume of $\mathrm{HS}$ to assess whether a precise volume of the solution was administered into the epidural space through the infusion pump. Although no complications were reported in this study, larger studies are needed to ensure reliability of the infusion pump.

In conclusion, our study suggests that performing DN with a continuous HS infusion is an effective way of reducing injection-related pain. Although the outcomes at the 3-month follow-up evaluation did not reveal any advantages, the continuous infusion of HS using an infusion pump in DN is less painful and less labor-intensive than is the repeated bolus injection of HS.

\section{ACKNOWLEDGMENTS}

The authors thank the Division of Statistics in the Medical Research Collaborating Center at Seoul National University Bundang Hospital for the assistance with the statistical 
analyses.

The infusion pump used in this study was supported by Hyun Medics Co., Wonju, Korea.

\section{CONFLICT OF INTEREST}

No potential conflict of interest relevant to this article was reported.

\section{ORCID}

Ho-Jin Lee, https://orcid.org/0000-0002-7134-5044

Jaewoo Lee, https://orcid.org/0000-0001-8100-7498

Yeon wook Park, https://orcid.org/0000-0001-6202-1673

Ho Young Gil, https://orcid.org/0000-0002-3438-9523

Eunjoo Choi, https://orcid.org/0000-0002-7002-3932

Francis Sahngun Nahm,

https://orcid.org/0000-0002-5900-7851

Pyung Bok Lee, https://orcid.org/0000-0003-0325-3356

\section{REFERENCES}

1. Lee F, Jamison DE, Hurley RW, Cohen SP. Epidural lysis of adhesions. Korean J Pain 2014; 27: 3-15.

2. Ross JS, Robertson JT, Frederickson RC, Petrie JL, Obuchowski N, Modic MT, et al. Association between peridural scar and recurrent radicular pain after lumbar discectomy: magnetic resonance evaluation. ADCON-L European Study Group. Neurosurgery 1996; 38: 855-61.

3. Igarashi T, Hirabayashi Y, Seo N, Saitoh K, Fukuda H, Suzuki $H$. Lysis of adhesions and epidural injection of steroid/local anaesthetic during epiduroscopy potentially alleviate low back and leg pain in elderly patients with lumbar spinal stenosis. Br J Anaesth 2004; 93: 181-7.

4. Robertson JT. Role of peridural fibrosis in the failed back: a review. Eur Spine J 1996; 5(Suppl 1): S2-6.

5. Trescot AM, Chopra P, Abdi S, Datta S, Schultz DM. Systematic review of effectiveness and complications of adhesiolysis in the management of chronic spinal pain: an update. Pain Physician 2007; 10: 129-46.

6. Racz GB, Heavner JE, Trescot A. Percutaneous lysis of epidural adhesions---evidence for safety and efficacy. Pain Pract 2008; 8: 277-86.

7. Helm li S, Benyamin RM, Chopra P, Deer TR, Justiz R. Percutaneous adhesiolysis in the management of chronic low back pain in post lumbar surgery syndrome and spinal stenosis: a systematic review. Pain Physician 2012; 15: E435-62

8. Waldman SD. Atlas of interventional pain management. 4th ed. Philadelphia, Elsevier Saunders. 2014, pp 568-76.

9. Birkenmaier C, Redeker J, Sievers B, Melcher C, Jansson V, Mayer-Wagner $S$. An evaluation of medications commonly used for epidural neurolysis procedures in a human fibroblast cell culture model. Reg Anesth Pain Med 2011; 36: 140-4.

10. Manchikanti L, Rivera JJ, Pampati V, Damron KS, McManus $\mathrm{CD}$, Brandon DE, et al. One day lumbar epidural adhesiolysis and hypertonic saline neurolysis in treatment of chronic low back pain: a randomized, double-blind trial. Pain Physician 2004; 7: 177-86.

11. Erdine S, Talu GK. Precautions during epidural neuroplasty. Pain Pract 2002; 2: 308-14.

12. Baraniuk JN, Ali M, Yuta A, Fang SY, Naranch K. Hypertonic saline nasal provocation stimulates nociceptive nerves, substance $\mathrm{P}$ release, and glandular mucous exocytosis in normal humans. Am J Respir Crit Care Med 1999; 160: 655-62.

13. Leffler AS, Kosek E, Hansson P. Injection of hypertonic saline into musculus infraspinatus resulted in referred pain and sensory disturbances in the ipsilateral upper arm. Eur $\mathrm{J}$ Pain 2000; 4: 73-82.

14. Bennell K, Hodges P, Mellor R, Bexander C, Souvlis T. The nature of anterior knee pain following injection of hypertonic saline into the infrapatellar fat pad. J Orthop Res 2004; 22: 116-21.

15. Choi EJ, Yoo YJ, Lee PB, Kim YC, Lee SC, Moon JY. A retrospective study to evaluate the effect of concentration of hypertonic saline on efficacy and safety of epidural adhesiolysis. Anesth Analg 2017; 124: 2021-9.

16. Jankovic D, Peng P. Regional nerve blocks in anesthesia and pain therapy: traditional and ultrasound-guided techniques. Cham, Springer. 2015, pp 655-70.

17. Kim J, Shin W. How to do random allocation (randomization). Clin Orthop Surg 2014; 6: 103-9.

18. Kim JH, Jung HJ, Nahm FS, Lee PB. Does improvement in epidurography following percutaneous epidural neuroplasty correspond to patient outcome? Pain Pract 2015; 15: 40713.

19. Seo H, inventor; Yoo J, assignee. Piezo-electric actuated infusion pump. Korea patents KR101127126B1. 2012 Mar 21.

20. Scarfone RJ, Jasani M, Gracely EJ. Pain of local anesthetics: rate of administration and buffering. Ann Emerg Med 1998; 31: $36-40$.

21. Cardoso MM, Carvalho JC. Epidural pressures and spread of $2 \%$ lidocaine in the epidural space: influence of volume and speed of injection of the local anesthetic solution. Reg Anesth Pain Med 1998; 23: 14-9.

22. Son WG, Jang M, Yoon J, Lee LY, Lee I. The effect of epidural injection speed on epidural pressure and distribution of solution in anesthetized dogs. Vet Anaesth Analg 2014; 41 : 526-33.

23. Usubiaga JE, Wikinski JA, Usubiaga LE. Epidural pressure 
Lee, et al / Continuous HS infusion vs. bolus injection || 205

and its relation to spread of anesthetic solutions in epidural space. Anesth Analg 1967; 46: 440-6.

24. Ho KY, Manghnani P. Acute monoplegia after lysis of epidural adhesions: a case report. Pain Pract 2008; 8: 404-7.

25. Graven-Nielsen T, Arendt-Nielsen L, Svensson P, Jensen TS. Quantification of local and referred muscle pain in humans after sequential i.m. injections of hypertonic saline. Pain 1997; 69: 111-7.

26. Lee $J H$, Lee SH. Clinical effectiveness of percutaneous adhesiolysis using Navicath for the management of chronic pain due to lumbosacral disc herniation. Pain Physician 2012; 15: 213-21. 